\title{
Very Deep Nulling Interferometers: limits and advantages
}

\author{
Daniel Rouan ${ }^{1}$ \\ ${ }^{1}$ LESIA-CNRS, Observatoire de Paris, 92195 Meudon cedex, France \\ email: daniel.rouan@obspm.fr
}

\begin{abstract}
Direct detection of a planet around a star by a nulling interferometer, requires to minimize as far as possible the stellar leaks due to the resolved angular size of the star. The original Bracewell configuration features a nulling function in $\theta^{2}$ which is insufficient in many cases. Several interferometric configurations have been proposed in order to improve the quality of the rejection with a nulling function $\theta^{n}$ with $2 \leqslant n \leqslant 6$. I proposed recently a method to build linear configurations of telescopes that achieve nulling function $\theta^{n}$ for any even value of $\mathrm{n}$, using the Prouhet-Thué-Morse sequence to select those telescopes where a $\pi$ phase shift is applied. In a first part, I recall the basis of this method and its generalization to $2 \mathrm{D}$ configurations and $1 \mathrm{D}$ arrays of non-identical telescopes, or even to configurations where the phase shift is not $\pi$. In a next step, I evaluate the efficiency of deep nulling interferometers in real world, i.e. when nulling is not perfect because of variations of distances or of phase shift between telescopes. I conclude that there is a clear advantage given by the highest order systems that keep a better nulling capability than conventional interferometers, even in severe conditions where parameters driving the nulling performance are highly fluctuating.
\end{abstract}

Keywords. Interferometry, Exoplanets, Nulling interferometry.

\section{Introduction}

There is a huge contrast $\left(10^{6-10}\right)$ between a star and a planet that orbits it, so that directly detecting the planet means cancelling as much as possible the stellar light. Bracewell, 1978 was the first to propose the concept of a nulling interferometer, where the light collected by two telescopes are coherently combined with however the trick that a $\pi$ phase shift is applied on one of the optical path. The system of fringes projected onto the sky shows a central dark fringe. If the star image is put on this central dark fringe, it disappears (actually, the stellar photons are all sent in the second output of the interferometer). If the planet is at the same time on a bright fringe, then it can be detected, in principle. The modulation of the fringes with respect to the angular distance is according to a $(1-\cos \theta)$ function, so that close to the axis the nulling is $\propto \theta^{2}$. If the interferometer has enough resolution (equivalently a long basis), as it should to separate a planet, then the angular diameter of the stellar disk is no longer small with respect to the fringe period and leaks of stellar light will contaminate the planet flux and severely limit the detection capability.

Several interferometric configurations have been presented in order to improve the quality of the rejection, especially in increasing the exponent of the term $\theta^{n}$ which gives the cancellation efficiency with respect to angular distance to the axis of the central fringe (e.g. Léger et al., 1995). The Angel's Cross (Angel, 1989), Oases configuration or Mariotti / Laurance (Darwin ESA project) configuration and others (Woolf, 1997) do fill this condition of a nulling with an exponent $>2$. In general, those configurations have been found through some trial method, but, as far as I know, no systematic method has been proposed to reach any given power of $\theta$. 
I presented recently (Rouan, 2003-a,Rouan, 2003-b) a method to define linear configurations of telescopes that provide a $\theta^{\mathrm{n}}$ nulling function for any even value of $n$. The principle is based on a remarkable property discovered by Prouhet (Prouhet, 1851) about a peculiar partition in two sets of the $\mathrm{N}=2^{L}$ first integers, done according to the Prouhet-Thué-Morse sequence. This property is such that, provided that to half of the N telescopes output a $\pi$ phase shift is applied, it is possible to cancel the (L-1) first terms of the developement in $\theta$ of the recombined amplitude, thus leading to the wished $\theta^{2 L}$ behaviour of the intensity. $1 \mathrm{D}$ configurations of identical telescopes is the basic configuration from which one starts to generalize the method for construction of $2 \mathrm{D}$ arrays still of identical telescopes -, then to build 1D and 2D arrays of non-identical telescopes and finally extend the theory to arrays where the phase shift is $2 \pi / \mathrm{n}$. The various steps of this approach are summarized in the first section.

However, this work would remain a rather formal exercise and more a mathematical trick than a true instrumental concept, if one would not go further. This is the object of section two to assess the actual performances of those Very Deep Nulling Interferometers (VDNI), when non perfect conditions of nulling are considered.

\section{Building Very Deep Nulling Interferometers}

\subsection{Prouhet-Thué-Morse sequence and Prouhet partition of the integers}

The Prouhet-Thué-Morse sequence is very easily built and can be considered as one of the simplest fractal objects. Let's start from the symbol "+". This is the first term of the series. Now take its inverse, "-", and concatenate it to the first element: this produces the second term of the series, i.e. "+ -". Let's redo the operation: we take the current term "+ -", and concatenate to it its complement, "- +": this leads to the third term, "+ - - +". The following terms are built the same way by concatenating the complement of the previous term:

$$
\begin{aligned}
& +--+-++- \\
& +--+-++--++-+--+ \\
& +--+-++--++-+--+-++-+--++--+-++- \text { etc. }
\end{aligned}
$$

One can note that: a) any term of the series never contains more than two identical digits (+ or -) that are juxtaposed (e.g. no +++ nor - - - -); b) periodic patterns never appear; c) the Lth term of the series contains $2^{L}$ digits (considering that the index of the first term is 1). Prouhet, 1851 had the idea to distribute the first $2^{L}$ integers into two sets according to the Prouhet-Thué-Morse sequence: the first set corresponds to integers that have the same rank as the $(+)$ in the sequence and the second set to those that have same rank as the $(-)$. This reads: $\mathcal{P}=\left[1,4,6,7,10,11,13,16, \ldots, o_{\mathrm{k}}, \ldots\right]$ and $\mathcal{M}=\left[2,3,5,8,9,12,14,15, \ldots, e_{\mathrm{k}}, \ldots\right]$

A property established by Prouhet, is that the sum of the elements of the two series are equal, but much more remarkably, the sum of the squares, of the cubes, etc. are also equal, and this up to the power L- 1 when considering the $2^{L}$ first integers. In a condensed form this can be written:

$$
\sum_{\mathrm{k}=0}^{2^{L-1}}\left(o_{\mathrm{k}}\right)^{p}=\sum_{\mathrm{k}=0}^{2^{L}-1}\left(e_{\mathrm{k}}\right)^{p}, \forall p<L .
$$

\section{2. $1 D$ nulling interferometers}

Let's consider an interferometer made with a set of $\mathrm{N}=2^{L}$ identical telescopes regularly aligned, with a distance $d$ between two adjacent telescopes, as illustrated on Fig. 1. If the telescopes are pointing in a direction $\theta$ (measured from the normal to the baseline), then the amplitude $a$ of the wave at the output of the recombiner is: 

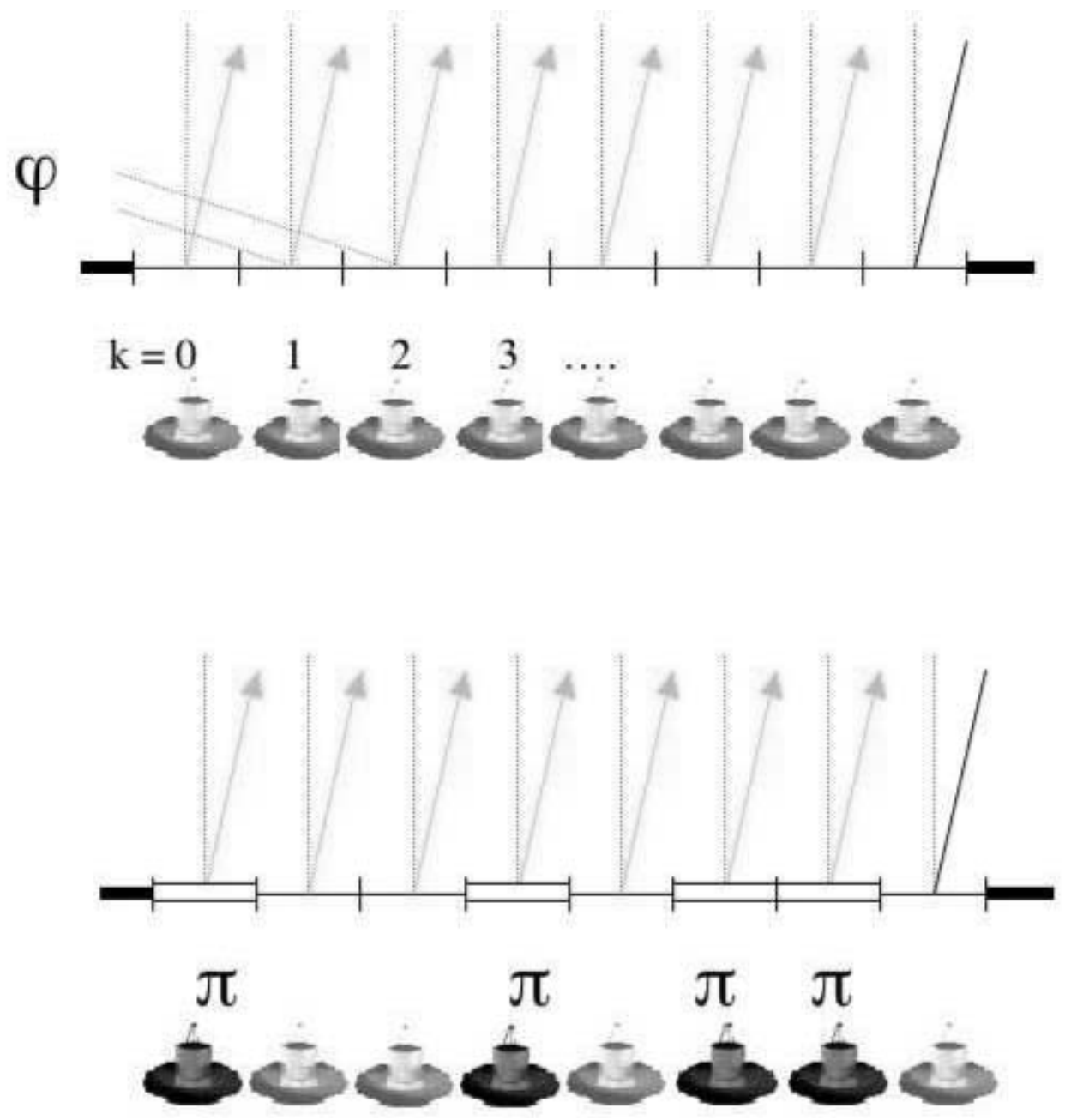

Figure 1. Top: configuration of a linear interferometer with equally spaced telescopes. Bottom: the fractal nulling interferometer, where some telescopes (the darker ones) experience a $\pi$ phase shift.

$$
a=\sum_{\mathrm{k}=0}^{\mathrm{N}} \exp (j \mathrm{k} \phi)
$$

Where $\phi$ is the phase difference between two consecutive telescopes: $\phi=2 \pi \theta d / \lambda$. Let's now add a $\pi$ phase shift on all telescopes whose rank is a "-" in the Prouhet series, in other word we change the sign of the amplitude. The previous relation becomes:

$a=a_{+}-a_{-}=\sum_{\mathrm{k} \in \mathcal{P}} \exp (j \mathrm{k} \phi)-\sum_{\mathrm{l} \in \mathcal{M}} \exp (j \mathrm{l} \phi)$

where $\mathcal{P}$ and $\mathcal{M}$ stand respectively for the "+" and "-" sets. Considering relatively small values of $\phi$, i.e. of $\theta$, one can develop each exponential in series of $(\mathrm{k} \phi)^{n}$, so that the two terms of the above expression become:

$$
\begin{aligned}
& a_{+}=\sum_{\mathrm{k} \in \mathcal{P}} 1+j \phi \times \sum_{\mathrm{k} \in \mathcal{P}} \mathrm{k}-\phi^{2} \times \sum_{\mathrm{k} \in \mathcal{P}} k^{2}-j \phi^{3} \times \sum_{\mathrm{k} \in \mathcal{P}} k^{3}+\ldots \\
& \text { and } \\
& a_{-}=\sum_{\mathrm{k} \in \mathcal{M}} 1+j \phi \times \sum_{\mathrm{k} \in \mathcal{M}} \mathrm{k}-\phi^{2} \times \sum_{\mathrm{k} \in \mathcal{M}} k^{2}-j \phi^{3} \times \sum_{\mathrm{k} \in \mathcal{M}} k^{3}+\ldots
\end{aligned}
$$

Thanks to the remarkable property found by Prouhet, i.e. $\sum_{\mathrm{k} \in \mathcal{P}} \mathrm{k}^{p}=\sum_{\mathrm{k} \in \mathcal{M}} \mathrm{k}^{p}$, the L-1 first terms of each series cancel out mutually, so that the wished property is 
reached: $a \propto \phi^{\mathrm{L}} \propto \theta^{\mathrm{L}}$, and the intensity has the behaviour: $I \propto \theta^{2 \mathrm{~L}}$. For instance, the interferometer shown in the lower part of Fig. 1 would produce a nulling function $\propto \theta^{6}$.

\subsection{Algebra of nulling interferometers}

2DVDN

Let's consider a square grid of telescopes where on any row or any column the sign of the output of each telescope is set following a Prouhet-Thué-Morse sequence - or its complement -. For instance the grid above would describe a $4 \times 4$ VDNI.

$$
\begin{array}{llll}
+ & - & - & + \\
- & + & + & - \\
- & + & + & - \\
+ & - & - & +
\end{array}
$$

The intensity varying as $\theta^{4 L}$, its nulling capability is in $\theta^{12}$. One can note that: a) the case $\mathrm{L}=1(2 \times 2$ telescopes) corresponds to the Angel's cross (Angel, 1989); $b$ ) the number of telescopes required to reach a given nulling exponent $\alpha$ (on the intensity) is the same in the $2 \mathrm{D}$ array and the $1 \mathrm{D}$ array cases: it is equal to $2^{\alpha / 2}$.

\section{VDNI with telescopes of different sizes}

One shows that by shifting by $\mathrm{k}$ steps each line with respect to the previous one, in the $2 \mathrm{D}$ array, and by summing algebraically the columns of the resulting matrix, one defines a 1D array of telescopes of different sizes which has the nulling power of the initial $2 \mathrm{~d}-$ array. For instance, the sheared $2 \mathrm{~d}$-array of the previous example

$$
\begin{array}{rrrrrrrrrr}
+1 & -1 & -1 & +1 & & & & & & \\
& & -1 & +1 & +1 & -1 & & & & \\
& & & -1 & +1 & +1 & -1 & & \\
& & & & & +1 & -1 & -1 & +1
\end{array}
$$

$\begin{array}{llllllllll}+1 & -1 & -2 & +2 & 0 & 0 & +2 & -2 & -1 & +1\end{array}$

leads to the $1 \mathrm{D}$ nulling configuration at the bottom line (0 means no telescope). One can also build $2 \mathrm{D}$ arrays from $1 \mathrm{D}$ arrays by simply having a matrix where the first column and the first line are any 1D VDNI and making the algebraic product at the intersection, as illustrated by he following example:

$$
\begin{array}{cccc}
+1 & -2 & +2 & -1 \\
-2 & +4 & -4 & +2 \\
+1 & -2 & +2 & -1
\end{array}
$$

\section{VDNI with phase shift different from $\pi$}

Finally, one shows that the Prouhet property can be extended to partitions of the first $\mathrm{n}^{L}$ integers in $\mathrm{n}$ sets rather than 2 , through some permutations at each step to build the terms of the series (Rouan, 2003-b). Provided that the phase shift for each group of telescopes becomes $2 \mathrm{k} \pi / \mathrm{n}$ instead of $\pi$, the nulling property in $\theta^{2 L}$ is as effective as in the bipartition.

\section{Comparison between VDNI interferometers in the real world}

We compare here the nulling properties of VDNI when a non perfect system is examined, i.e. when distances and phase are fluctuating, so that nulling is no longer constant. Indeed the question of the nulling stability is an issue since the associated noise tends to be the most important contributor in the present studies of space interferometers (Lay, 2004; Chazelas, 2005). For this comparison, I considered only 2D square arrays of the 

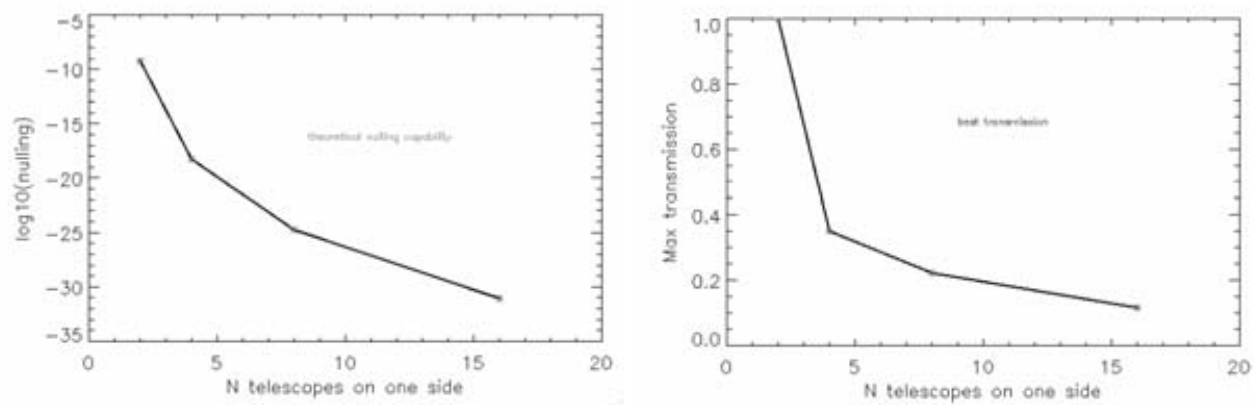

Figure 2. Left: theoretical nulling capability of the four first 2D square VDNI vs the number of telescopes on one side; Right: best transmission of the VDNI.

basic type: $2 \times 2,4 \times 4,8 \times 8,16 \times 16$. I assumed that the total surface of telescopes was the same: either few large telescopes or many small telescopes. I have in mind the TBC assumption that in terms of mission cost, this could be equivalent because the mass production of identical small telescopes should be rather cheap (e.g. $64 \times 25 \mathrm{~cm}$ telescopes vs $4 \times 1 \mathrm{~m}$ telescopes). Simulations were done for an Earth-Sun system: this means that the distance between telescopes is such that the projected first maximum of transmission is at $1 \mathrm{AU}$ and that the star diameter to consider for leaks calculation is .01 AU. Two causes of nulling degradation were considered in this preliminary exercise: $a$ ) the distances between telescopes are not constant and the rms relative variation is $\delta d / d=10^{-3}$; b) there is an imperfect $\pi$ phase shift with an rms fluctuation of .01 radians. Fig. 2, 3, and 4 summarize the results of this study.

First, in terms of absolute performances (perfect nulling configuration), the nulling capability of VDNI is indeed excellent and far beyond the required factor of $10^{10}$ for an Earth detection, since, for instance, it reaches $10^{-32}$ for the $16 \times 16$ array (Fig. 2-a). However the maximum transmission of the planet is decreasing with the order, it is $12 \%$ for the $16 \times 16$ pattern, while it is $100 \%$ for the $2 \times 2$ one (Fig. 2 -b).

The most interesting results is the one giving the nulling performance when fluctuations of distances and of phase are introduced: Fig.3a-b shows that there is a clear improvement when using, for a given level of fluctuation, larger arrays: for instance, even with a very severe phase fluctuation of $.5^{\circ} \mathrm{rms}$, the nulling capability can be maintained at a fairly good level with a $16 \times 16$ array, with a gain of 85 over the $2 \times 2$ array. If one looks at the detail of the behavior with time of the nulling performance (Fig. 4), it clearly appears that the larger the array, the lesser the amplitudes of fluctuations, and especially the big ones (note the logarithmic scale of Fig. 4): for instance in the case of the $2 \times 2$ array, the nulling factor can vary by as much as a factor of 2000 , while it is only a factor of 370 for the $16 \times 16$ array. One can logically suspect some averaging effect to explain this robustness of the nulling efficiency in the higher order VDNI.

Since the stability of the nulling efficiency is clearly a concern in the design of space nulling interferometer, it seems thus that considering the VDNI concept to improve this performance deserves some attention. More detailed estimates are obviously required.

\section{Conclusion}

Reaching a good nulling on starlight is mandatory for detecting terrestrial planets. A method to design a nulling interferometer that provides a central null depth of any given even power of $\theta$ has been given previously. It is based on a property of the Prouhet-ThuéMorse sequence, and allows to build 1D interferometers where to half of the telescopes 

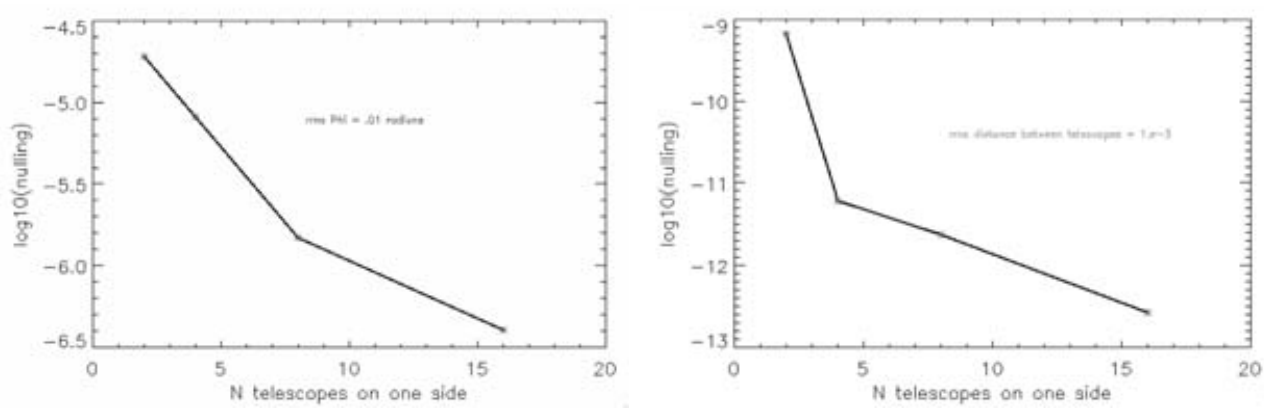

Figure 3. Left: nulling capability of the four first 2D square VDNI when distance between telescopes fluctuates, with $\mathrm{rms} \delta d / d=10^{-3}$; Right: same but when $\pi$ phase shift fluctuates, with $\sigma_{\phi}=1 \mathrm{deg}$.

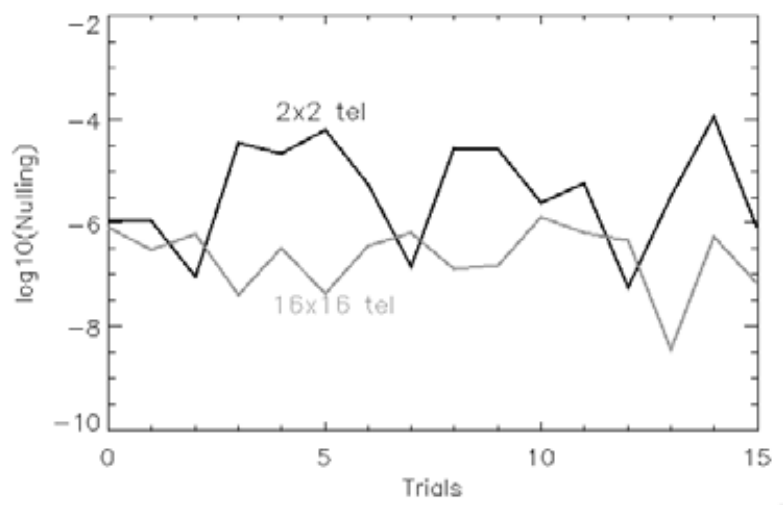

Figure 4. Exemple of nulling stability as a function of time for $2 \times 2$ and $16 \times 16$ patterns.

is applied a $\pi$ phase shift. From this basic pattern, one can then build 2D or other $1 \mathrm{D}$ patterns, by combining in one dimension or two dimensions those building bricks. In a second step I estimate how those VDNI behave when the basic interferometric parameters (distances and phase) fluctuate, as this happens in the real world. I show that the higher the order of the VDNI, the more robust they are with respect to nulling performances, beating largely conventional nulling interferometers of lower orders. This is especially true when very severe phase variations are introduced. This robustness is likely the result of some averaging effects. Especially the nulling stability noise, which is a very serious concern in the design of space interferometers, is largely reduced with a high order VDNI. This type of interferometer may be a serious alternative to more conventional design, if indeed the nulling stability noise remains the main concern in presently studied missions. For a given collecting surface, the increasing number of telescopes could be compensated, in terms of cost, by the mass production of identical small telescopes.

\section{References}

Angel, R., 1989, in The Next Generation Space Telescope, p. 81

Bracewell, R.N., 1978, Nature 274, 780

Chazelas, B., Brachet, F., Ollivier, M., Mennesson, B., et al., 2005, A\&A accepted

Lay, O.P., 2004, Applied Optics, 43, 6100-6123

Léger, A., Mariotti, J.-M., Menesson, B., et al., 1996, Icarus 123, 249 
Prouhet, E., 1851, Mémoire sur quelques relations entre les puissances des nombres. in: Comptes Rendus des Séances de l'Académie des Sciences

Rouan, D., 2003, Proceedings of the Conference on Towards Other Earths: DARWIN/TPF and the Search for Extrasolar Terrestrial Planets, 22-25 April 2003, Heidelberg, Germany. Edited by M. Fridlund, T. Henning, ESA SP-539, Noordwijk, Netherlands, p. 565 - 568

Rouan, D., 2004, From Fractals to Exoplanets: building ultra-nulling interferometers, EAS Publications Series, 12, 21

Woolf, N.J. \& Angel, J.R.P., 1997, "Planet Finder Options I: New Linear Nulling Array Configurations", in "Planets beyond the solar system and the next generation of space missions". ASP Conference Series, ed. D. Soderblom 

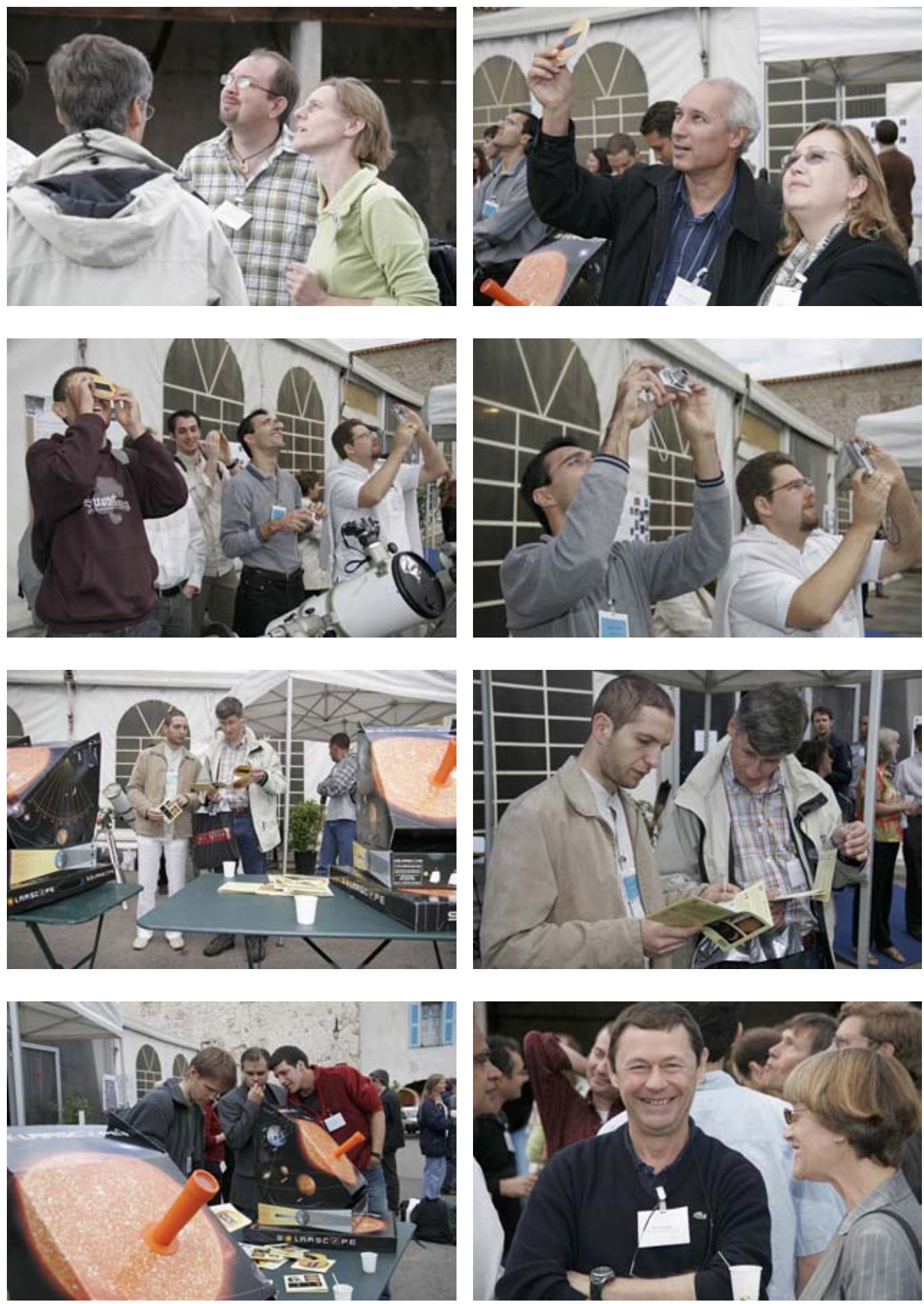

All photographs: Laurent Thareau [1.thareau@free.fr]. 